\title{
Is postural tachycardia syndrome in the head or in the heart? And other updates on recent autonomic research
}

\author{
Mitchell G. Miglis ${ }^{1} \cdot$ Srikanth Muppidi ${ }^{1}$
}

Received: 2 May 2017/ Accepted: 4 May 2017/Published online: 13 May 2017

(C) Springer-Verlag Berlin Heidelberg 2017

\section{Postural tachycardia syndrome and anxiety: the head or the heart?}

Anxiety in patients with postural tachycardia syndrome (POTS) is a controversial topic. Most autonomic clinicians agree that patients with POTS frequently have symptoms of anxiety or depression, and the prevalence of suicidal ideation in this population is noteworthy [8]. However, many of the clinical symptoms of POTS overlap with those of anxiety, and studies failed to establish causation [9].

Owens and colleagues, in an article published in the March 2017 issue of Autonomic Neuroscience, add novel insight to this growing body of literature [7]. They assessed the prevalence of "somatic hypervigilance" (i.e., the ability to perceive internal sensations like palpitations, gastrointestinal upset, or temperature intolerance) in different disorders, and how one's sensitivity to these sensations, i.e., interoception, may enhance anxiety. The degree to which a person is sensitive to interoceptive signals has been linked to emotional experience, and those who are more sensitive to fluctuations in their body's internal feedback system may be more prone to anxiety. In addition, discrepancies between expected and actual homeostatic signals may also contribute to anxiety, something the authors describe as "interoceptive prediction errors". The authors administered a series of rating scales, including an anxiety sensitivity index and a body vigilance scale, to 21 patients with POTS, 20 patients with vasovagal syncope (VVS), 20 patients with essential hyperhidrosis (EH, a

Srikanth Muppidi

muppidis@stanford.edu

1 Department of Neurology, Stanford Neurosciences Health Center, 213 Quarry Road, Palo Alto, CA 94304, USA disorder highly associated with anxiety), and 23 age-matched healthy controls. They asked all subjects to perform a heartbeat perception test designed to assess interoceptive sensitivity. This involved silently counting each heartbeat during a period of pseudorandom duration, at rest and during periods of supine isometric exercise and supine cold pressor testing (designed to increase sympathetic activation). They then compared the patients' estimates to their actual EKG-derived heart rate. Body vigilance scores were increased in all patient groups, with the highest results in POTS patients. POTS, VVS and EH patients all underestimated the number of heartbeats during heartbeat counting tasks, indicating interoception prediction errors, and patients with POTS committed greater errors during tests of sympathetic activation.

These results are in keeping with a previous publication by Ramesh Khurana in Clinical Autonomic Research [2], in which the heartbeat perception test was similar patients with POTS and controls, indicating lack of superior interoceptive ability in POTS, though patients did have greater ability to discriminate the type of palpitation (thumping, pounding, racing, etc.) when compared to controls. As in the study by Owens and colleagues, palpitation severity was increased on provocative tests of sympathetic activation, suggesting that palpitations in POTS are mediated by sympathoexcitatory stimuli, favoring the concept of visceral hypersensitivity.

These studies on interoception and somatic vigilance illustrate why autonomic medicine is perfectly poised to investigate the connections between the mind and the body, and we look forward to future investigations unveiling the actual pathophysiology of POTS.

Invited Commentary (Dr. Ramesh Khurana): "Owens and colleagues have extended our observations in a larger cohort with a modified experimental design. They 
applied a heartbeat perception test at baseline and during three physiological stimuli: isometric handgrip test, cold pressor test, and head-up tilt. They confirmed our finding that interoceptive accuracy was not superior at baseline or during sympathetic stimulation. In addition, the authors assessed the anxiety sensitivity index (ASI), body vigilance scale (BVS), self-consciousness scale, and childhood traumatic events scale and discovered that patients with POTS have higher global BVS and ASI scores, indicating somatic hypervigilance. Both studies support the concept that abnormal processing/interpretation of somatic and external sensations may contribute to POTS".

\section{Predicting the future: REM sleep behavior disorder and neurodegeneration}

It is well established that idiopathic REM sleep behavior disorder (iRBD), or RBD without clinical signs of neurodegenerative disease, is a risk factor of a neurodegenerative CNS $\alpha$-synucleinopathy [Parkinson disease (PD), dementia with Lewy bodies (DLB), and multiple system atrophy (MSA)]. It is now apparent that the vast majority of patients with iRBD will eventually develop PD, DLB or MSA within their lifetimes [10].

In a study published in the April 18th issue of Neurology [4], the authors followed 43 patients with video polysomnography-confirmed iRBD over the course of 4 years. They administered a battery of rating scales including the Montreal Cognitive Assessment scale (MoCA) and the Scale for Outcomes in Parkinson DiseaseAutonomic (SCOPA-AUT). All patients underwent olfactory testing with the Sniffin Sticks 16-item test (SS-16) and all had dopamine transporter single positive emission computerized tomography (DAT) scans. Patient with iRBD had a rate of phenoconversion of $10 \%$ per year. Patients with abnormal DAT scans had up to a threefold greater risk of developing parkinsonism over the 4-year period. Strikingly, the authors found no correlation with anosmia and phenoconversion, in contrast to other studies. The authors justify this by explaining that some of the odors in the SS16 were culturally unfamiliar to the Chinese patients.

Of interest to autonomic practitioners, the authors found that the presence of autonomic symptoms was associated with a higher phenoconversion risk. The autonomic symptoms reported on the SCOPA-AUT that most correlated with phenoconversion were gastrointestinal symptoms, genitourinary symptoms, orthostatic symptoms, and excessive sweating. In the Natural History Study of the Synucleinopathies (summarized in a previous issue of Clinical Autonomic Research [6]), predictors of phenoconversion to $\mathrm{PD} / \mathrm{DLB}$ in those with autonomic failure included RBD with impaired olfactory function, whereas predictors of phenoconversion to MSA included RBD with preserved olfaction [1].

Many patients with iRBD have some degree of autonomic impairment, and many patients with $\alpha$-synucleinopathies and autonomic impairment also have RBD. There is limited data on the role of objective autonomic testing as a more sensitive predictor than subjective rating scales in these patients, and we await the results of larger studies to help answer these question and help pave the way for the neuroprotective trials.

\section{A novel sudomotor testing method: will it make a difference?}

Sudomotor testing is an integral part of autonomic testing to determine pre- or post-ganglionic sudomotor dysfunction. Most autonomic centers currently perform quantitative sudomotor axonal reflex testing (QSART). Some centers also offer thermoregulatory sweat testing (TST). Among the various sudomotor tests that are routinely available, QSART provides total sweat volume, while other testing such as silastic imprinting provides a spatial distribution of sweat production in a defined area.

In April 2017, the Journal of the Peripheral Nervous System published online the work by Loavenbruck and colleagues detailing a new method designed to obtain temporal and spatial sweat production measures, including total sweat volume, rate of sweat production in individual sweat glands, and the number of secreting sweat glands per area [5]. In their technique, sweat production was stimulated by iontophoresis of $0.5 \%$ pilocarpine at four sites (foot, medial calf above the ankle, distal medial thigh and dorsum of the hand), not entirely different from the typical sites used with the current QSART protocol. But, here is the difference: after iontophoresis, sites were dried and painted with iodine. The researchers then mounted a camera capable of taking serial images over a $2.25 \mathrm{~cm}^{2}$ area once every second. With the serial images, the total sweat volume, density of the sweat glands in the chosen area and the rate of sweat production were calculated. They assessed sweat volumes in 178 healthy controls at the 4 designated sites and showed that the results of this new technique follow the same pattern as those in prior QSART studies in healthy controls. Women tended to have lower sweat volumes, and distal sites tended to have lower volumes than proximal sites. In addition, they also tested this method in 20 patients with neuropathy at the distal 2 sites in the lower limb, and all sweat measures were lower in patients with neuropathy when compared to healthy controls. 
This novel testing technique appears to be rigorous and reliable and the results follow an expected pattern in both healthy controls and patients. However, it is unlikely that the authors achieved one of their goals, namely to create a comprehensive, easy-to-use sweat testing technique. The technique is like QSART in that it employs chemical iontophoresis followed by a measurement of sweat output. The novel addition in this protocol seems to be the camera technology; however, this addition is likely to add longer processing times to the sweat testing protocol. The authors do point to an additional feature of recognizing focal areas of reduced sweat production within the recorded area, which is currently not available with QSART technology. It is possible that this technique is more sensitive due to a greater degree of granular information on sweat gland distribution and sweat production, and thus may be employed in research studies to assess small fiber or peripheral autonomic dysfunction. However, practical limitations may limit its use in routine clinical practice. When measuring sudomotor function, finding the balance between highly accurate but complicated techniques and bedside fast tests (e.g., the spoon test [3]) remains a significant research challenge.

Acknowledgements We thank Dr. Ramesh Khurana for his comment.

\section{Compliance with ethical standards}

Conflict of interest The authors declare that they have no competing interests.

Funding None.

\section{References}

1. Kaufmann H, Norcliffe-Kaufmann L, Palma JA, Biaggioni I, Low PA, Singer W, Goldstein DS, Peltier AC, Shibao CA, Gibbons CH, Freeman R, Robertson D, Autonomic Disorders C (2017) Natural history of pure autonomic failure: a United States prospective cohort. Ann Neurol 81:287-297

2. Khurana RK (2014) Visceral sensitization in postural tachycardia syndrome. Clin Auton Res Off J Clin Auton Res Soc 24:71-76

3. Khurana RK, Russell C (2017) The spoon test: a valid and reliable bedside test to assess sudomotor function. Clin Auton Res Off J Clin Auton Res Soc 27:91-95

4. Li Y, Kang W, Yang Q, Zhang L, Zhang L, Dong F, Chen S, Liu J (2017) Predictive markers for early conversion of iRBD to neurodegenerative synucleinopathy diseases. Neurology 88:1493-1500

5. Loavenbruck AJ, Hodges JS, Provitera V, Nolano M, Wendelshafer-Crabb G, Kennedy WR (2017) A device to measure secretion of individual sweat glands for diagnosis of peripheral neuropathy. J Peripher Nerv Syst. doi:10.1111/jns.12212

6. Muppidi S, Miglis MG (2017) Is pure autonomic failure an early marker for Parkinson disease, dementia with Lewy bodies, and multiple system atrophy? And other updates on recent autonomic research. Clin Auton Res Off J Clin Auton Res Soc 27:71-73

7. Owens AP, Low DA, Iodice V, Critchley HD, Mathias CJ (2017) The genesis and presentation of anxiety in disorders of autonomic overexcitation. Auton Neurosci Basic Clin 203:81-87

8. Pederson CL, Brook JB (2017) Health-related quality of life and suicide risk in postural tachycardia syndrome. Clin Auton Res Off J Clin Auton Res Soc 27:75-81

9. Raj V, Raj SR (2017) Postural tachycardia syndrome and suicide: what we don't know. Clin Auton Res Off J Clin Auton Res Soc 27:69-70

10. Schenck CH, Boeve BF, Mahowald MW (2013) Delayed emergence of a parkinsonian disorder or dementia in $81 \%$ of older men initially diagnosed with idiopathic rapid eye movement sleep behavior disorder: a 16-year update on a previously reported series. Sleep Med 14:744-748 